\title{
The Role of Self-Efficacy in Achieving Health Behavior Change
}

\author{
Victor J. Strecher, PhD, MPH \\ Brenda McEvoy DeVellis, PhD \\ Marshall H. Becker, PhD, MPH \\ Irwin M. Rosenstock, PhD
}

The concept of self-cfficacy is recciving increasing recognition as a predictor of health behavior change and maintenance. The purpose of this article is to facilitate a clearer understanding of both the concept and its relevance for health education rescarch and practice. Self-efficacy is first defined and distinguished from other related concepts. Next, studies of the self-efficacy concept as it relates to health practices are examined. This review focuses on cigarette smoking. weight control, contraception, alcohol abuse and exercise behaviors. The studies reviewed suggest strong relationships between self-efficacy and health behavior change and maintenance. Experimental manipulations of self-efficacy suggest that efficacy can be enhanced and that this enhancement is related to subsequent health behavior change. The findings from these studies also suggest methods for modifying health practices. These methods diverge from many of the current, traditional methods for changing health practices. Recommendations for incorporating the enhancement of self-efficacy into health behavior change programs are made in light of the reviewed findings.

\section{INTRODUCTION}

Bandura's Social Learning Theory attempts to predict and explain behavior using several key concepts; among these are incentives, outcome expectations and selfefficacy expectations. Although all are important, the concept of self-efficacy expectations is of particular relevance to health education. However, despite the critical role it appears to play in the initiation and maintenance of behavior change, self-efficacy has received relatively little attention in the health education research and practice literatures. The purpose of this article is to facilitate a clearer understanding of both the concept and its relevance for health education research and practice.

\footnotetext{
Victor J. Strecher is Assistant Professor, Department of Health Education, University of North Carolina.

Address reprint requests to Victor J. Strecher, $\mathrm{PhD}, \mathrm{MPH}$, Department of Health Education, School of Public Health, 311 Rosenau Hall 201 H. Chapel Hill, North Carolina 27514.

Brenda McEvoy DeVellis is Associate Professor for the Department of Health Education, University of North Carolina.

Marshall H. Becker is Professor and Chair. Department of Health Behavior and Health Education. University of Michigan.

Irwin M. Rosenstock is Professor, Department of Health Behavior and Health Education, University of Michigan.
} 
We will begin by defining the concept, and then describe ways in which perceptions of self-efficacy develop and exert their influence on behavior. In addition, we will distinguish self-efficacy from related concepts. This first section draws heavily upon four works by Bandura ${ }^{1-4}$ in which he presented the theory and conceptualization of self-efficacy and provided empirical support for his conclusions. The next portion of the article reviews research that has examined the self-efficacy construct in the context of attempts to alter health-related behaviors. This section illustrates the current stateof-the-art in self-efficacy research, and identifies empirical evidence regarding applications of self-efficacy theory to health education programs, examining how perceptions of self-efficacy have been manipulated to influence health-related behaviors. In the final section, research and practice implications of the first two sections are discussed. Current models of health education programming are described in light of self-efficacy theory, and recommendations are offered for incorporating the enhancement of selfefficacy into health education programs.

\section{THE THEORETICAL FRAMEWORK}

Bandura ${ }^{2}$ outlines the role of self-efficacy in the paradigm of a person engaging in a behavior that will have a consequent outcome.

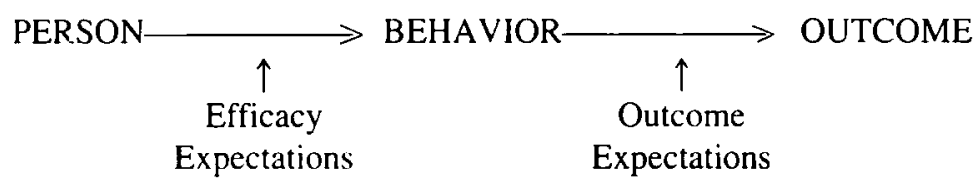

According to this paradigm, behavior change and maintanance are a function of (1) expectations about the outcomes that will result from one's engaging in a behavior; and (2) expectations about one's ability to engage in or execute the behavior. Thus, "outcome expectations" consist of beliefs about whether a given behavior will lead to given outcomes, whereas "efficacy expectations" consist of beliefs about how capable one is of performing the behavior that leads to those outcomes. It should be emphasized that both outcome and efficacy expectations reflect a person's beliefs about capabilities and behavior-outcome links. Thus, it is these perceptions, and not necessarily "true" capabilities, that influence behavior. In addition, it is important to understand that the concept of self-efficacy relates to beliefs about capabilities of performing specific behaviors in particular situations; ${ }^{5}$ self-efficacy does not refer to a personality characteristic or a global trait that operates independently of contextual factors. ${ }^{2}$ This means that an individual's efficacy expectations will vary greatly depending on the particular task and context which confronts him/her. It is therefore inappropriate to characterize a person as having "high" or "low" self-efficacy without reference to the specific behavior and circumstance with which the efficacy judgment is associated.

Bandura argues that perceived self-efficacy influences all aspects of behavior, including the acquisition of new behaviors (e.g., a sexually-active young adult learning how to use a particular contraceptive device), inhibition of existing behaviors (e.g., decreasing or stopping cigarette smoking), and disinhibition of behaviors (e.g., resuming sexual activity after a myocardial infarction). Self-efficacy also affects people's choices of behavioral settings, the amount of effort they will expend on a task, and 
the length of time they will persist in the face of obstacles. Finally, self-efficacy affects people's emotional reactions, such as anxiety and distress, and thought patterns. Thus, individuals with low self-efficacy about a particular task may ruminate about their personal deficiencies rather than thinking about accomplishing or attending to the task at hand; this, in turn, impedes successful performance of the task.

According to Bandura, ${ }^{1-4}$ efficacy expectations vary along dimensions of magnitude, strength and generality. Each of these dimensions has important implications for performance, and each implies slightly different measurement procedures. "Magnitude" refers to the ordering of tasks by difficulty level. Persons having low-magnitude expectations feel capable of performing only the simpler of a graded series of tasks, while those with high-magnitude expectations feel capable of performing even the most difficult tasks in the series. "Strength" refers to a probabilistic judgement of how certain one is of one's ability to perform a specific task. ${ }^{6}$ A two-step measurement procedure assures that, where appropriate, both dimensions will be tapped. First, individuals are presented with a list of performance activities reflecting various difficulty levels and are asked to designate those tasks they believe they can accomplish at that time. Then, for each designated task, they rate the strength of their belief on a 10 unit interval scale ranging from 10-100.

The third dimension, "generality," concerns the extent to which efficacy expectations about a particular situation or experience generalize to other situations. For example, the beliefs of post myocardial infarction patients about their endurance capabilities generated during supervised exercise testing may or may not generalize to unsupervised exercising at home.

\section{Development of Efficacy Expectations}

\section{Sources of Efficacy Information}

Efficacy expectations are learned from four major sources. The first, termed performance accomplishments, refers to learning through personal experience where one achieves mastery over a difficult or previously feared task and thereby enjoys an increase in self-efficacy. Performance accomplishments attained through personal experience are the most potent source of efficacy expectations.' Successive mastery over tasks required to engage in a behavior helps the person to develop and refine skills. In addition, it fosters development of a repertoire of coping mechanisms to deal with problems encountered.

The second source is vicarious experience, which includes learning that occurs through observation of events and/or other people. These events/people are referred to as "models" when they display a set of behaviors or stimulus array that illustrates a certain principle, rule or response. 'For example: A man who feels vulnerable to a heart attack may fear the consequences of exercising after watching a $T . V$. drama in which a male character has a myocardial infarction following exercise; a woman who hopes to quit smoking but observes a friend's difficulty in abstaining from cigarettes may come to believe that she herself will never be able to quit. On the other hand, observing a model master situations which have been feared or seen as difficult can enhance one's own expectations of mastery. In order for modeling to affect an ob- 
server's self-efficacy positively, however, it is important that the model can be viewed as overcoming difficulties through determined effort rather than with ease, and that the model be similar to the observer with regard to other characteristics (e.g. age, sex). Additionally. modeled behaviors presented with clear rewarding outcomes are more effective than modeling with unclear or unrewarded outcomes.

Verbal persuasion constitutes the third source of efficacy expectations. This method is quite familiar to all health educators who have exhorted patients to perservere in their cfforts to change behavior.

Finally, one's physiological state provides information that can influence efficacy expectations. Bandura'.2 has noted that because high physiological arousal usually impairs performance, people are more likely to expect failure when they are very tense and viscerally agitated. For example: People who experience extremely sweaty palms. a racing heartbeat and trembling knees prior to giving a talk find that their self-efficacy plummets; to someone just beginning an exercise program. fatigue or mild aches and pains may be mistakenly interpreted as a sign of physical inefficacy. ${ }^{3}$

\section{Appraisal of Efficacy Information}

Appraisal of efficacy information is important because data from various sources do not automatically influence perceived efficacy. Instead, information is attended to, weighted, and interpreted in ways that mediate its impact on efficacy expectations. ${ }^{4}$ An example of an attentional factor is selective self-monitoring. People may differ in their tendencies to attend to and remember various aspects of performance. Thus, some people may focus on the positive aspects of their performance and overlook the negative ones; such overestimation can have negative consequences, because these individuals may attempt tasks beyond their capabilities. On the other hand, those who dwell on their failures may underestimate what they can do, and be reluctant to try new behaviors (resulting in lost opportunities for skill and efficacy development).

The impact of efficacy information can also be influenced by how it is weighted. Individuals may self-monitor positive and negative aspects of performance accurately but tend to discount the importance of one aspect. For example, people with low selfesteem may tend to discount positive efficacy information. Another example pertains to the relative weight one gives to efficacy information communicated by people who vary in degree of credibility: Information from a highly-credible person will have a greater impact on efficacy expectations than will messages from a less credible person.

The final type of mediator. interpretation, is best explained by attribution processes. An achievement will enhance self-efficacy only if it is attributed to one's own ability or skill and not to external, chance, or temporary factors. ${ }^{2}$ Certain characteristics of the situation and task can influence the types of attributions that will be made when someone experiences success. For example. when success is achieved with minimal effort it is apt to be attributed to one's ability which. in turn, fosters a sense of selfefficacy. On the other hand, the same degree of success achieved through a great deal of effort is less likely to result in attributions to ability. and consequently, self-efficacy is less likely to be enhanced. Bandura notes that people's self-efficacy also can affect the types of attributions they make in situations. For example. people with a high sense of self-efficacy may attribute occasional failures to chance or to some temporary condition and thus maintain their success orientation. ${ }^{6}$ 


\section{Related Concepts}

Appropriate operationalization and measurement of self-efficacy depend on conceptual clarity. It is therefore important to understand the differences between selfefficacy and a number of other concepts that are sometimes linked and frequently confused with it. This confusion occurs. in part, because the personality traits, states, and processes that these concepts represent can influence efficacy expectations or be influenced by them. However, this does not mean that any of these concepts are equivalent to self-efficacy.

"Health Locus of Control" refers to a generalized expectation about whether one's health is controlled by one's own behavior or by forces external to oneself. ${ }^{7}$ Health is an outcome, while self-efficacy focuses on beliefs about the capacity to undertake behavior(s) that may or may not lead to desired outcomes (such as health). Bandura ${ }^{2}$ illustrates the importance of the distinction between locus of control and self-efficacy by noting that the conviction that outcomes (e.g. good health) are determined by one's own action can have any number of effects on self-efficacy and behavior. For example, people who view their health as personally determined but who believe they lack the skills needed to carry out the behaviors that would result in good health would experience low self-efficacy and approach those activities with a sense of futility.

"Self-esteem" refers to a liking and respect for oneself that has some realistic basis. Thus, self-esteem is concemed with an evaluation of self-worth, while self-efficacy relates to an evaluation of specific capabilities in specific situations. ${ }^{6}$ Bandura highlights the distinction between the two concepts by pointing out that people can have high self-efficacy for a task from which they derive no self-pride (e.g., being able to brush one's teeth well) or have low self-efficacy for a task but have no loss of self-worth (e.g., not being able to ride a unicycle). However, he observes that people often try to develop self-efficacy in activities that give them a sense of self-worth, so that the two concepts are frequently intertwined.

"Anxiety and Depression." The concept of anxiety is not part of either the definition or the measurement of self-efficacy: self-efficacy scales ask people to assess performance capabilities, not whether they can perform activities nonanxiously. However, anxiety results when people see themselves as ill-equipped to deal with potentially injurious events. Anxiety, in turn, may influence expectations of efficacy. Bandura 2 has demonstrated that high levels of physiological arousal inhibit self-efficacy and, as a result, performance (see previous discussion of sources of efficacy expectations). Anxiety can result from perceived inefficacy in the face of potentially injurious events, while depression occurs when people feel inefficacious at getting highly-valued outcomes. ${ }^{+}$Bandura also notes the frequent co-occurence of anxiety and depression, and explains that this happens when people are confronted with situations where obtaining a valued outcome. such as getting a job. will forestall future aversive events.

"Coping." According to Lazarus and Folkman's" conceptualization, coping is viewed as a process; like Bandura, they downplay notions of coping that emphasize generalized dispositional concepts such as self-esteem. "Secondary appraisal" is defined as an individual's evaluation of what might and can be done in the face of a threat or challenge. During this complex process, a person takes into account (either consciously or unconsciously): (1) Which coping strategies are available; (2) the likelihood that some strategy will accomplish the expected outcome; and (3) whether one can apply that strategy or strategies effectively." Clearly the second part of this process is similar 
to the notion of outcome expectation, while the third is equivalent to self-efficacy. Thus, within Lazarus and Folkman's framework, efficacy expectations are part of secondary appraisal.

"Learned Helplessness." Learned helplessness refers to cognitive, affective and motivational deficits that result from exposure to uncontrollable events. The attributional reformulation of learned helplessness suggests that there are two types of helplessness: personal and universal. Personal helplessness occurs when an individual lacks the requisite controlling response in a situation but believes this response is available to others; i.e., the individual believes that although he or she cannot control the outcome, others in the same situation could. In the case of universal helplessness, the individual still lacks the requisite response but believes that the outcome is independent of any response he/she or others could make. ${ }^{10}$ Abramson et al. ${ }^{10}$ view this distinction between personal and universal helplessness as consistent with Bandura's distinction between efficacy and outcome expectancies. Low efficacy and high outcome expectations characterize personal helplessness while low efficacy and low outcome expectations characterize universal helplessness.

\section{REVIEW OF STUDIES}

In this section, research employing the concept of self-efficacy is examined, with the studies grouped into the following general health practice areas: smoking; weight control; contraceptive behavior; alcohol abuse; and exercise. Other health practice areas (e.g., compliance with medical regimens; dietary change unrelated to weight control) were not included because of a lack of research using the efficacy concept. The review is also limited to research conducted after publication of Bandura's seminal article and book on self-efficacy. ${ }^{1.2}$

Table I summarizes the studies that have investigated the self-efficacy construct. Note that the studies are grouped by behavior under investigation, beginning with cigarette smoking and followed by weight control, contraceptive behavior, alcohol abuse and exercise. The first column in the Table specifies the health-related behavior examined. The second column indicates name(s) of author(s). Study design and sample are included in column three: type of study design (retrospective or prospective); type of method; number of subjects; selected characteristics of the sample and the composition of the sample. The studies have been categorized into three different methodologies: (1) Survey studies examining self-efficacy levels of subjects who vary according to a particular health-related behavior (which we term "survey" in the Table); (2) studies examining the effect of a standard behavior change program on questionnaire-measures of self-efficacy (which we term "survey" in the Table); and (3) experiments which deliberately manipulate self-efficacy as a treatment variable (which we term "experiment" in the Table). Column four presents the results for each study. The final column includes notes about the association of self-efficacy with other concepts, and about the generalizability of self-efficacy.

\section{Cigarette Smoking}

A model of smoking cessation developed by Pechacek and Danaher ${ }^{11}$ identifies outcome and efficacy expectations as predictors in the initiation and maintenance of 


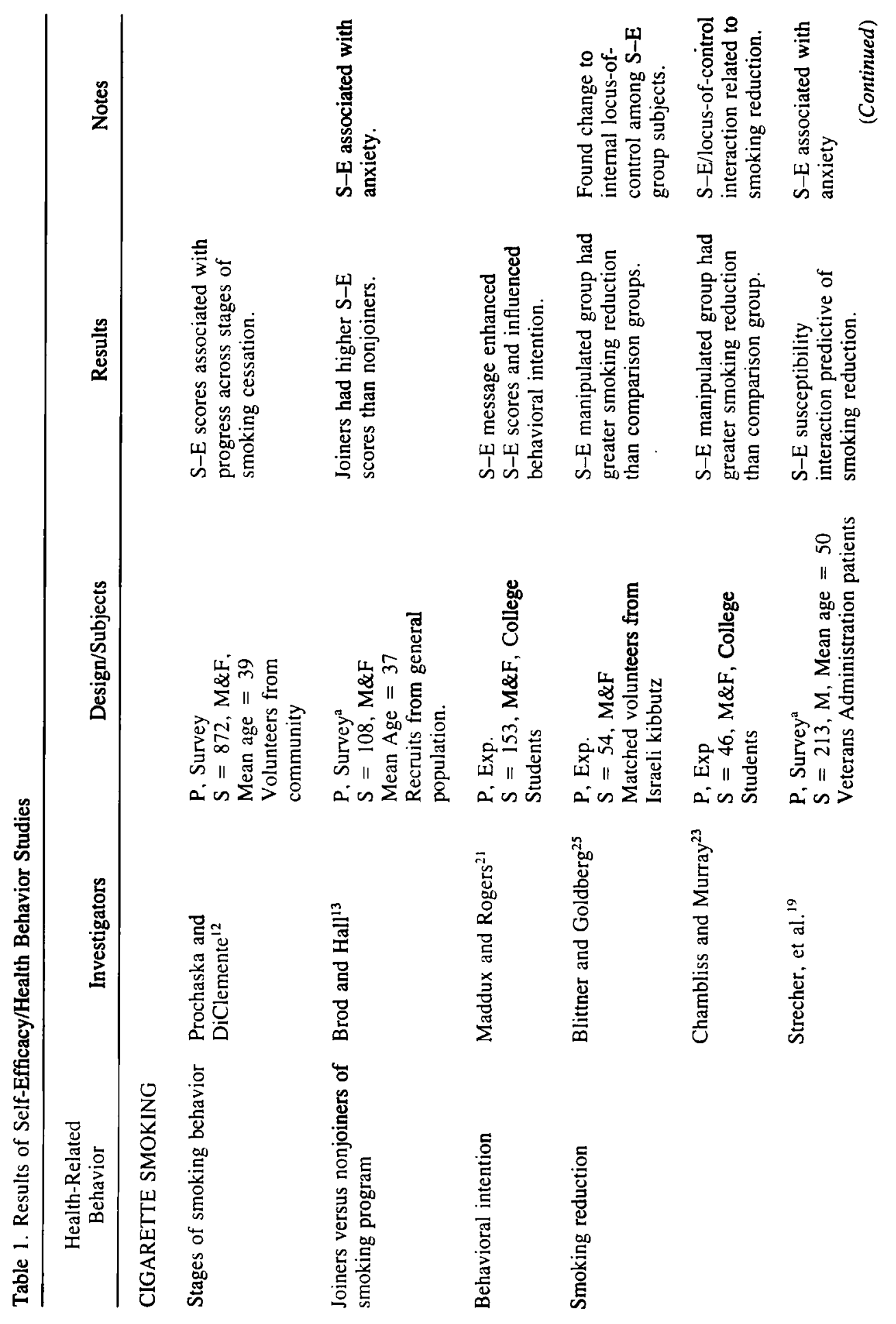




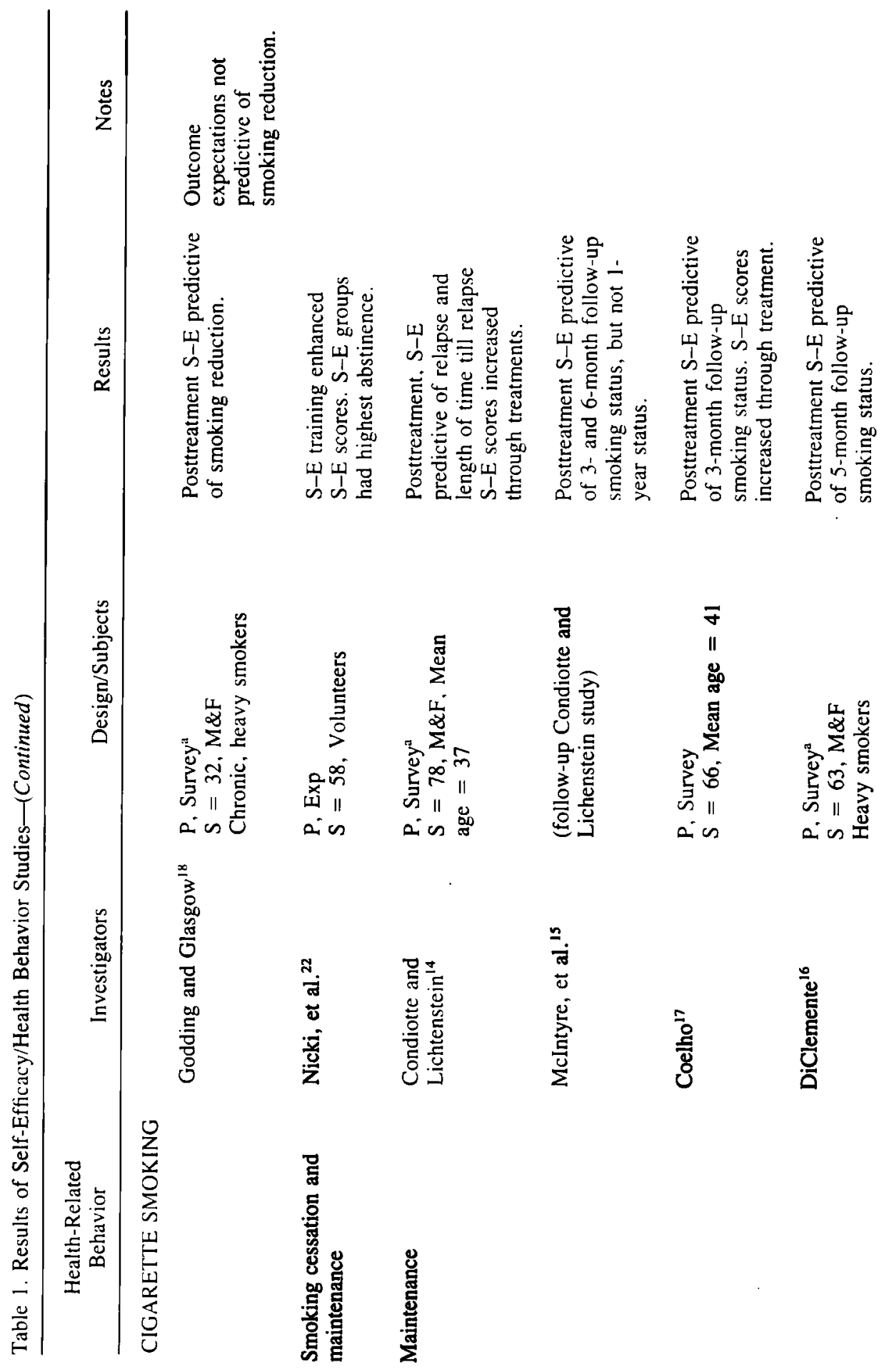




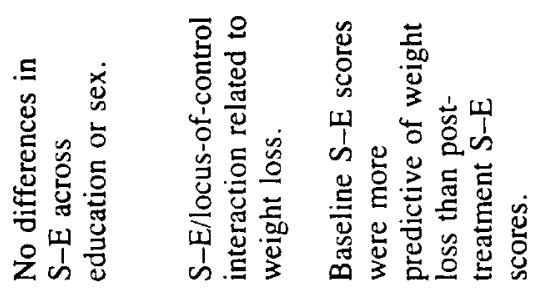
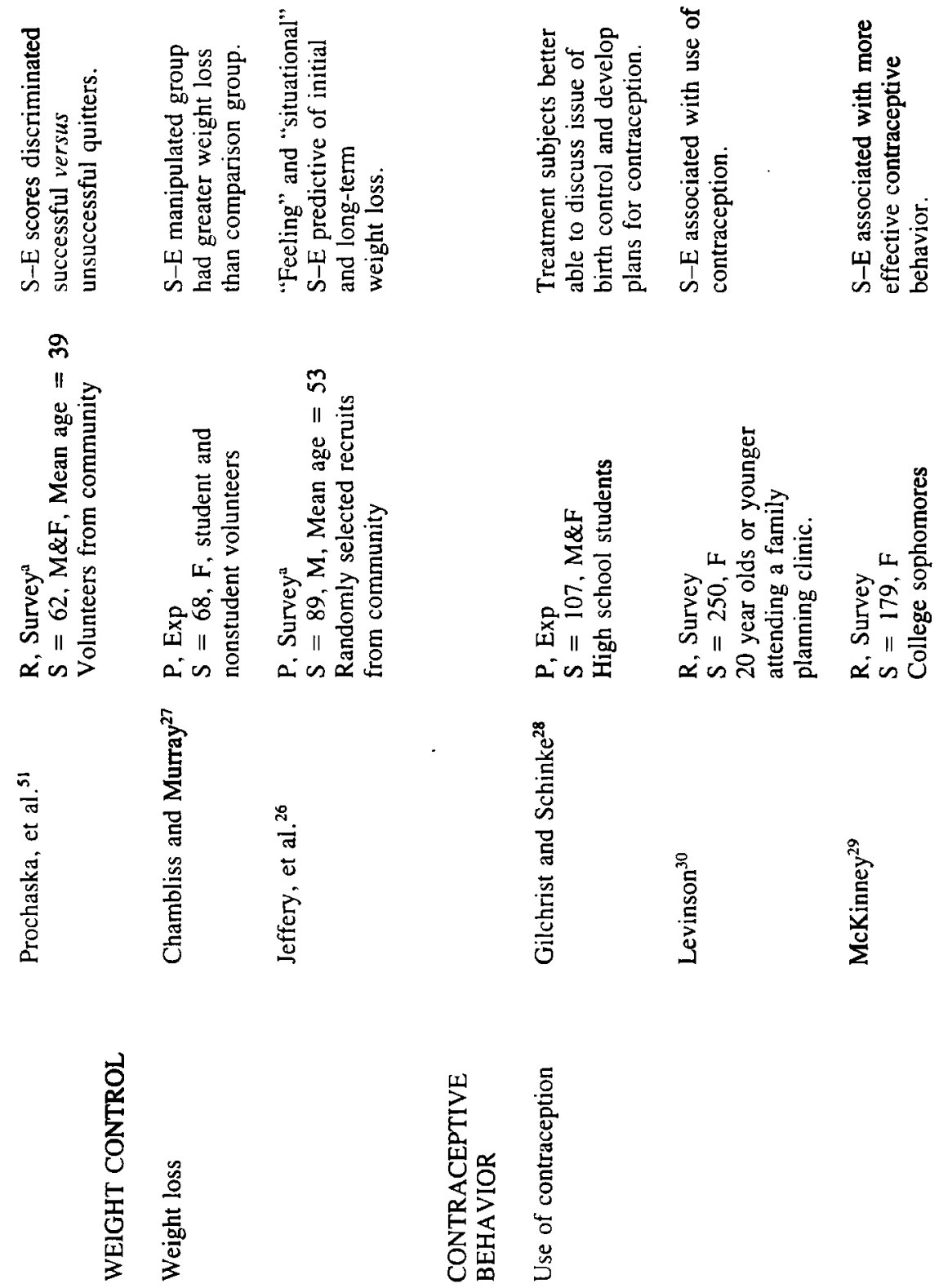


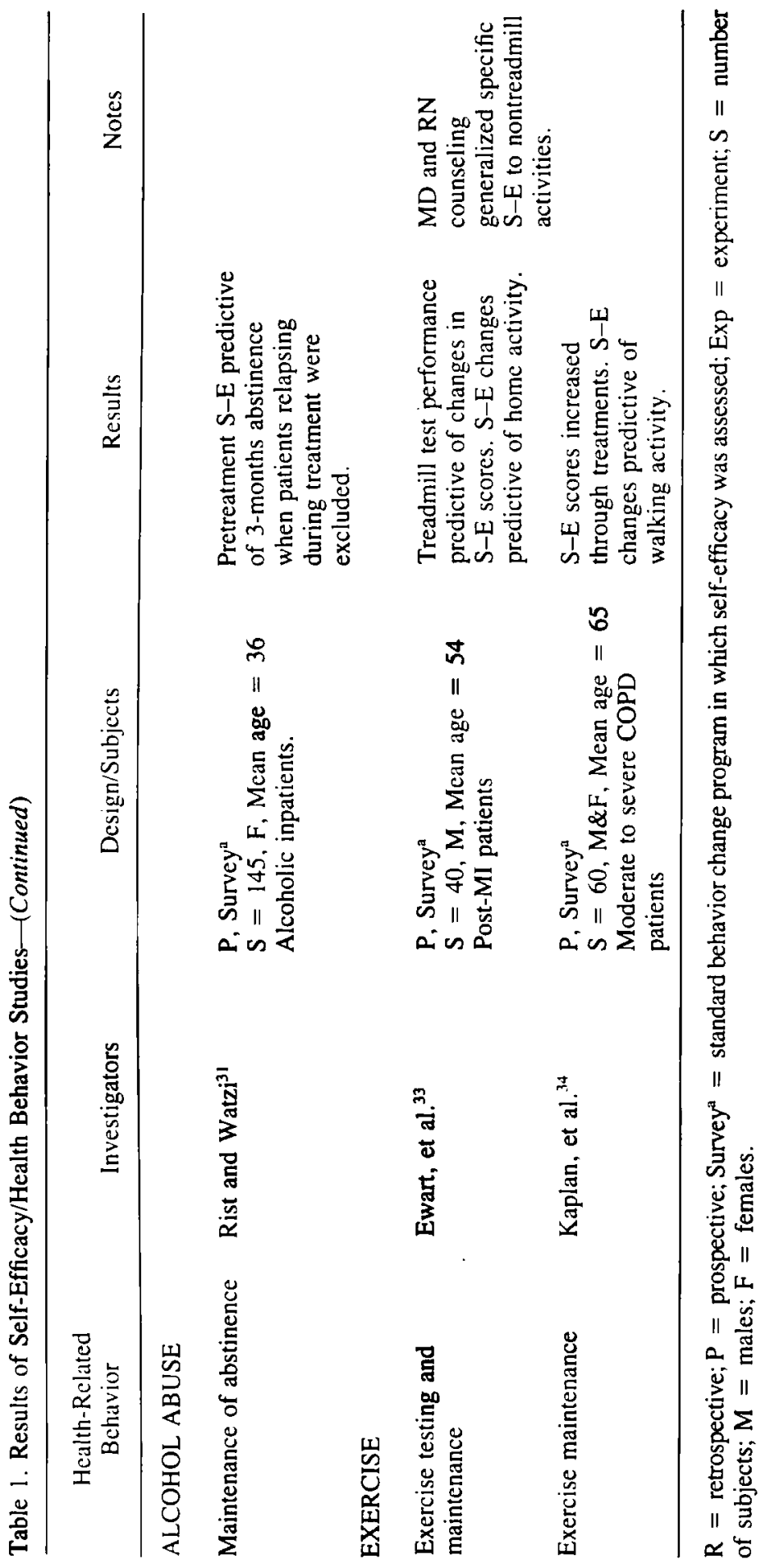


cessation. The authors suggest that outcome expectations mediate initial motivation to quit smoking, while efficacy expectations influence both cessation and maintenance processes. Studies using survey methodology tend to support this model of smoking behavior change. Prochaska and DiClemente ${ }^{12}$ examined 872 "ever-smokers," categorizing them into five stages: precontemplation (smokers with no intention of quitting smoking); contemplation (smokers seriously considering quitting); action (recent quitters); maintenance (quitters for at least 6 months); and relapse (those who failed to quit). Self-efficacy, and a number of other psychosocial constructs, were used as predictors of the stages into which subjects were categorized at baseline and at sixmonth follow-up. Self-efficacy was associated with movement from precontemplation, contemplation or action stages. Consistent with Prochaska and DiClemente's findings, Brod and Hall ${ }^{13}$ found that subjects electing to join a smoking cessation program reported higher levels of efficacy than did nonjoiners.

In prospective research, Condiotte and Lichtenstein ${ }^{14}$ found increased self-efficacy appraisals as a result of completing either of two different smoking-cessation programs. Low ratings of self-efficacy following treatment were highly predictive of relapse, and of length of time before relapse. McIntyre, et al. ${ }^{15}$ followed the same smokers from the Condiotte and Lichtenstein study over one year, finding initial low efficacy ratings still predictive of relapse at six months, but weakening to non-significance by one year following treatment. Similar results have been found by DiClemente ${ }^{16}$ and Coelho. ${ }^{17}$ These studies did not measure outcome expectations, though it is likely that such expectations would already be strong in subjects motivated to enter smoking-cessation programs."

A recent study by Godding and Glasgow ${ }^{18}$ examined the effects of efficacy as well as outcome expectations on subsequent control of smoking behavior, measured by nicotine content of cigarettes smoked, the amount of each cigarette smoked, and the number of cigarettes smoked. Posttreatment efficacy ratings were predictive of reductions in each of these variables at a six-month follow-up. Outcome expectations did not appreciably add to the explanatory power of the self-efficacy measure. However, the results are limited by the small sample size $(N=32)$, which did not permit testing for an interaction between outcome and efficacy expectations. Also, it is likely that enrollees in a program to control smoking would have high outcome expectations, and that there would be little variance in responses to a measure of this concept.

A prospective study by Strecher, et al. ${ }^{19}$ examining outcome expectations (measured by perceived susceptibility to health hazards of continued smoking) and a proximal measure of self-efficacy (anticipated difficulty in refraining from smoking in those situations identified by Condiotte and Lichtenstein ${ }^{1+}$ ) found no bivariate relationship between the two constructs or between either construct and outcomes in a self-help smoking-cessation program. However, an interaction was found between outcome and efficacy measures with respect to smoking reduction. Subjects with high pretreatment outcome and efficacy expectations exhibited the highest overall smoking reduction three months following treatment, while subjects with high outcome but low efficacy expectations exhibited the lowest overall level of smoking reduction. This particular group (high outcome/low efficacy) conforms with the characterization of personal "learned helplessness." 11.20 The self-help program, designed to enhance self-efficacy through gradual skills development, was found to influence success in this learned helplessness group. The high outcome/high efficacy group, however, exhibited no difference in smoking reduction between treatment and control (both groups did better 
than groups exhibiting any other combination of outcome and efficacy expectations). This may have occurred because self-efficacy was already high and the treatment was redundant for these subjects. A similar interaction effect between outcome and efficacy expectations on intention to quit smoking was found by Maddux and Rogers. ${ }^{21}$

Nicki and colleagues ${ }^{22}$ examined the usefulness of self-efficacy training in combination with a "nicotine fading" program (i.e., switching to progressively lower nicotinecontent cigarettes) as compared to self-instructional training in combination with the same nicotine fading program. Subjects in the self-efficacy/nicotine fading group received information on the usefulness of self-efficacy in quitting smoking. Over the course of treatment. they were then asked to choose successively more difficult situations in which to refrain from smoking (using a situational rating scale similar to DiClemente's $\left.{ }^{16}\right)$. Successive accomplishments were interpreted in the treatment as increasing competency and mastery over cigarette smoking.

Self-instructional training included an explanation of "self-talk" and how it may affect cigarette smoking. Appropriate self-talk instructions were taught to subjects. who were instructed to develop and apply similar patterns of thought to real-life situations. Results found self-efficacy training, in combination with nicotine fading, to be significantly more effective than nicotine fading alone, or than nicotine fading in combination with the self-instructional training program. A fourth group, which included self-efficacy training, self-instructional training and nicotine fading was no more effective than the self-efficacy training and nicotine fading combination. Also, increases in reported self-efficacy over the course of treatment paralleled overall decreases in rate of smoking and in rate of nicotine intake.

Chambliss and Murray ${ }^{23}$ manipulated perceptions of efficacy in a group of smokers enrolled in a treatment program by administering a placebo pill to subjects. Half of the subjects were later informed that the pill was a fake and that their success up to that point could be attributed to their own competence (the other subjects were left to continue believing in the efficacy of the pill). Prior to the experiment, subjects were given Rotter's Locus of Control Scale ${ }^{24}$ and were subsequently classified as "internals" (having generalized expectations that outcomes are contingent on personal behavior) or "externals" (having generalized expectations that outcomes are unrelated to personal behavior). The researchers found a significant main effect of the efficacy manipulation in reducing cigarette smoking. However, this effect was largely due to a significant interaction between locus of control and the efficacy manipulation; the self-efficacy manipulation was effective only among subjects with an internal locus of control. These results suggest that self-efficacy may be more important among those who feel that their personal actions control health outcomes.

In another experimental manipulation of self-efficacy, Blittner and collegues ${ }^{25}$ administered a battery of psychological tests to a group of volunteers in a smoking-cessation program. Although they did not use the term "self-efficacy," they essentially manipulated it by admitting only a portion of the subjects into the cessation program, telling them that they were admitted in preference to other volunteers because their tests had demonstrated great potential for being able to quit smoking. The subjects were actually selected at random, and the tests were not used as predictors of success. Another group received the same psychological tests, but were told that they were selected at random, and that the tests were administered as part of a different study. Both groups then received a smoking-cessation treatment program. Average smoking frequency was reduced by $67 \%$ fourteen months following treatment for the efficacyenhanced group, and by $35 \%$ for the comparison group. Locus of control was measured 
prior to experimental manipulation, but the concept was treated by the researchers as a dependent variable as opposed to a moderating variable (as was the case in the Chambliss and Murray ${ }^{2.3}$ study). Subjects in the efficacy-enhanced group exhibited a significant shift toward an internal locus of control from pre- to post-treatment.

In summary, the self-efficacy construct has been examined in the area of smoking and smoking cessation through a variety of survey and experimental studies. This research has been extremely fruitful in delineating the role of efficacy expectations vis-a-vis outcome expectations as well as other psychosocial constructs, such as stress and locus of control. Ratings of self-efficacy were found to discriminate active quitters from continued smokers, joiners of smoking cessation programs from non-joiners and successful from unsuccessful short- and long-term quitters who have participated in smoking cessation programs. Experimental manipulations of self-efficacy suggest that efficacy may be enhanced and this enhancement is related to subsequent smoking cessation and reduction.

\section{Weight Control}

Like smoking cessation, weight control is generally considered a desirable (though often difficult to achieve) goal-and one barrier to its achievement may be a lack of self-efficacy with regard to engaging in the required behaviors. In a prospective study of factors related to weight loss one and two years following a behavioral treatment program, Jeffery and collegues ${ }^{26}$ assessed efficacy expectations at pretreatment, posttreatment. and one-year follow-up. In this study, self-efficacy was divided into "emotion state" efficacy, which described ratings of confidence in ability to refrain from eating during various emotional states, and "situational" efficacy, which reflected confidence in ability to abstain in various situations such as visiting friends or watching television. High pretreatment levels of emotional state and situational efficacy were significantly associated with initial and with long-term (both one- and two-year) weight loss. Posttreatment and follow-up efficacy expectations were not as powerful in predicting long-term weight loss, although posttreatment situational efficacy was associated with initial and one-year weight loss and posttreatment emotional state efficacy was associated with initial weight loss.

In an experiment similar to their smoking-cessation study discussed earlier, Chambliss and Murray ${ }^{27}$ manipulated efficacy expectations by offering a placebo medication (along with a standard weight control program) to overweight women and then reattributing successful weight control to personal abilities. This reattribution was accomplished by informing half of the subjects about the placebo medication and congratulating them on their efforts. The other subjects remained in the "drug efficacy" condition. Prior to the experiment, subjects were given Rotter's Locus of Control Scale. ${ }^{24}$ The investigators found no bivariate associations between locus of control or self-efficacy and subsequent weight change. However, a significant interaction between locus of control and self-efficacy was found: subjects in the self-efficacy group who also had an internal locus of control experienced the highest weight loss, while those in the self-efficacy group with an external locus of control experienced a slight gain in weight. The reverse was true for the drug-efficacy group: externals were more successful than internals. These results are very interesting as they suggest further refinements of the efficacy-behavior linkage. 


\section{Contraceptive Behavior}

Effective, consistent use of contraception involves engaging in a set of skills which include frank communication about birth control between partners, dealing with partner demands, and acquisition and use of specific devices. ${ }^{28}$ In retrospective survey studies, McKinney ${ }^{29}$ found that women's perceptions of personal skill and competence at using contraception were related to more effective contraceptive behavior, while Levinson ${ }^{30}$ found that teenage women's confidence in their ability to use contraception in situations where a contraceptive priority was in conflict was positively associated with contraceptive use.

In an attempt to build efficacy through a cognitive-bchavioral treatment, Gilchrist and Schinke ${ }^{28}$ developed an intervention for adolescents which presented factual material on reproduction and birth control together with skills training and practice. Skills training included participation in role playing situations where discussion of birth control is initiated, methods of contraception are acquired, and unacceptable demands are refused. Prior to role playing exercises, relaxation exercises were given to the students.

As a result of this cognitive-behavioral intervention, students showed marked improvements in efficacy ratings of their own abilities to use birth control, exhibited more effective contraceptive problem solving abilities, and had greater intentions to use contraception at next intercourse than did a group of students not receiving the intervention. Unfortunately, follow-up data on actual contraceptive use or rates of pregnancy were not ascertained. If, however, skills and intention are the first steps to effective use of contraception, this study offers a useful efficacy-based strategy for improving contraceptive behavior.

\section{Alcohol Abuse}

To our knowledge, only one published study to date has examined the effect of self-efficacy in the treatment of alcohol addiction. ${ }^{31}$ In this study, females addicted to alcohol were enrolled in a three-month inpatient treatment program which involved social-skills training. Prior to and following the training, subjects rated themselves according to how difficult they felt it would be to refuse a drink under a number of social situations. Although not a direct estimate of self-efficacy, the authors' findings suggest that perceived personal difficulty in coping with social situations closely reflects a generalized efficacy expectation with respect to maintaining abstinence. For subjects overall, ratings of perceived personal difficulty in refusing alcohol diminished over the course of treatment and were also found to predict success in the program.

\section{Exercise}

Although the association between efficacy expectations and athletic performance has been examined in a number of studies (e.g., Weinberg. et al. ${ }^{32}$ ), only two studiesboth examining patient populations-have examined the effect of efficacy expectations on compliance with exercise regimens. In a study of men with clinically uncomplicated myocardial infarction. Ewart and collegues ${ }^{3.3}$ found that changes in efficacy scores as a result of treadmill exercise testing predicted both the duration and intensity of 
subsequent self-reported home activity. Self-efficacy assessments were correlated with subsequent performance on the treadmill test. which, in turn, predicted subsequent changes in self-efficacy. The researchers also found that counseling from a physician and nurse was useful in causing the effects of self-efficacy to be generalized from arcas specifically related to the treadmill test (running; walking; climbing stairs) to less-related activities (e.g., lifting; sexual intercourse).

In a study of patients with chronic obstructive pulmonary disease, Kaplan, et al. ${ }^{34}$ randomly assigned subjects to one of five experimental groups, including three exercise treatment (to increase walking) groups and two control groups. Subjects in each of the three treatment groups increased their walking activity in comparison to those in the control groups; this increase was associated with increases in perceived efficacy for walking. The study also found that efficacy expectations specifically related to the target behavior were most predictive of subsequent performance of that behavior.

\section{DISCUSSION}

\section{Self-Efficacy in Health Behavior Research}

For all health-related areas studied in this review, self-efficacy appears to be a consistent predictor of short- and long-term success. In experimental studies. manipulations of self-efficacy have proven consistently powerful in initiating and maintaining change, supporting Bandura's assertion that efficacy expectations reflect a person's perceived, rather than actual, capabilities, and that it is these perceptions and not one's true abilities that often influence behavior. Studies measuring the effect of a standard behavior change program on self-efficacy found overall increases in efficacy over the course of treatment, and found efficacy to be related to short and long term success as a result of the program. Survey studies of self-efficacy reviewed suggest strong associations between self-efficacy and progress in health behavior change and maintenance.

Although the self-efficacy framework addresses both efficacy and outcome expectations, it was surprising to find so few studies examining outcome expectations in conjunction with efficacy expectations. Outcome expectations are a major component of value-expectancy models of health behavior (e.g., the Health Belief Model ${ }^{35.36}$ ). Perceptions of susceptibility to a particular illness, and of the benefits of taking recommended preventive actions could both be considered expectations of outcome. These expectations may play the largest role in influencing initial motivation and decision to change a health practice. ${ }^{11.19}$ Health practices which are not particularly difficult to modify but whose perceived consequences are uncertain may largely depend on outcome expectations. An example of this might be compliance with medication to control hypertension, which, for many, is not a difficult behavior, but one which may have questionable outcomes. Studies have found that outcome expectations, such as perceived susceptibility to hypertension-related illnesses or perceived benefits of taking the medication, are associated with hypertension medication compliance. ${ }^{36}$

Where the health practice is believed to lead to desired consequences but the change is difficult to make, self-efficacy considerations are probably paramount. This is often the case among smokers, many of whom wish to quit, but feel unable to do so. Finally, for more difficult to change practices of uncertain consequences, both outcome and 
efficacy expectations may be required to explain the behavior change process. This might be the case in the modification of certain dietary habits, such as increasing the intake of fiber to reduce cancer risk. The link between fiber intake and cancer risk is still not well known or believed by much of the American public and modifications in nutrition behavior are often difficult to make for any extended period of time. In this case, both outcome and efficacy expectations would require careful examination.

Although the studies reviewed generally conceptualized self-efficacy as perceived capabilities of performing specific behaviors. operational measures of self-efficacy varied considerably. As mentioned carlier. Bandura suggests a two-step approach to measuring self-efficacy: asking the subject whether he/she believed a particular behavior could be accomplished. and then. for each designated task. asking the subject to rate the strength of his/her belief. In many of the studies examined, this method was incorporated into a unified set of measures which included both strength and magnitude. In most studies, subjects were asked how confident they would feel in performing the target behavior in different situations or mood states where the ability to perform the behavior might vary.

Kirsch ${ }^{37.38}$ has argued that eliciting an estimate of confidence in performing behaviors which are physically within reach of a person's abilities may not be measuring selfefficacy so much as willingness to perform the behavior. Levels of confidence for behaviors which are physically possible to perform, such as quitting smoking or simple exercise, may therefore be mediated by an often implicit (though variable) expectation of reward or punishment (an outcome expectation). For in-depth analysis of this measurement issue, the reader is referred to the ongoing conceptual and methodological debate between Kirsch ${ }^{37.38}$ and Bandura. ${ }^{39}$

Consistent with the assertions of Bandura ${ }^{1.2}$ results of one study reviewed ${ }^{34}$ suggest that measures of self-efficacy must be specifically related to the behavior in question. Effects of an intervention designed to increase walking behavior were mediated by efficacy expectations for walking. Among efficacy measures for other behaviors, only those measures which were closely related to walking (e.g., climbing) were found to mediate subsequent walking behavior. Although it is probably important to assess efficacy as it relates to the specific behaviors in question, recent evidence ${ }^{\text {t0 }}$ suggests that measuring ability to engage in the target behavior under varying conditions may not be as important as was first thought. Psychometric examination of a frequentlyused questionnaire assessing confidence in ability to refrain from smoking under a wide range of situations and mood states ${ }^{14}$ found a large, general unidimensional factor that could be tapped from a much smaller number of items. Further research is required to understand the heuristics underlying generalization from one task-related efficacy expectation to another, as well as from one situation to another.

In studies where other psychosocial constructs are also examined (e.g. locus of control, anxiety). self-efficacy consistently emerges as a distinct and powerful predictor of behavior. Other findings suggest that certain psychosocial constructs interact with or influence self-efficacy. The finding of Chambliss and Murray ${ }^{23.27}$ that self-efficacy manipulation was effective only for those with an internal locus of control suggests that the importance of self-efficacy may, in large part, depend on perceived influence in controlling outcomes. The cross-sectional association between anxiety and selfefficacy found in a number of studies examined ${ }^{13.19}$ may reflect Bandura"s assertion that physiological arousal influences self-efficacy. It is also likely that low self-efficacy is a source of anxiety. Interactions between locus of control, anxiety. self-efficacy, and other psychosocial constructs must be more carefully examined and delineated. 


\section{Using Self-Efficacy in Practice}

One of the strengths of the self-efficacy framework is its direct applicability to the practice of modifying health behaviors. Given the evidence from the studies reviewed, as well as results from general behavior change studies, suggestions about an overall strategy for enhancing sclf-eficacy in practice would include first breaking the complexities of the target behavior into components which are relatively easy to manage. This requires a carcful examination of the target behavior and identification of specific aspects of the behavior which call for skills development. Specific behaviors must then be arranged in a series so that they may be consecutively mastered, with initial tasks being casier than subsequent tasks. As accomplishments lcading to the overall target behavior progress in this step-wise manner, encouragement should be given to: (1) Demonstrate the person's relative progress toward the target behavior; and (2) attribute previous accomplishments to the person's own abilities. Relative progress may also be demonstrated by charting progress over the course of the change process. Lapses in behavior should be treated as opportunitics to analyze and subsequently control the factors which caused the lapse. ${ }^{41}$

The present review found support for this strategy from studies which manipulated self-efficacy in field or in laboratory conditions. Developing awareness of specific situations where efficacy may be low and rehearsing desired behavior in these situations appears to enhance efficacy. ${ }^{2 x}$ Other methods of enhancing efficacy include relaxation training to reduce anxiety during the behavior change process ${ }^{28.34}$ and verbal reinforcement to enhance efficacy ${ }^{22.23 .25 .27}$ The work of Ewart and collegues ${ }^{33}$ suggests that counseling from a credible source may be effectively used to generalize specific task-related efficacy expectations to other behaviors.

The self-efficacy model suggests modes of treatment that diverge from many current health behavior-change strategies. Bartlett" states that "self-efficacy theory provides a theoretical buttress for the notion of an "activated" patient" (p.547). However, many current models of health behavior change attempt to "deactivate" individual responsibility for changing. The "medical model" of treatment holds that the individual is not responsible for the solution to his/her problem. ${ }^{4.3}$ Within this model, the individual is compliant to the judgements of the expert and the expert, in turn, takes responsibility for the outcome of the individual. This applies to medically-based treatments to modify behavior (e.g.. drug or surgical treatments) as well as to less traditional modes of treatment (e.g., hypnosis or accupuncture).

In Alcoholics Anonymous (AA), alcoholics take responsibility for their problem, but are also required to acknowledge inability to control alcoholism without the help of a higher power and the members of $\mathrm{AA} .{ }^{+3}$ In removing the responsibility for control from the individual, self-efficacy may be diminished. ${ }^{+4}$ Weisz and others ${ }^{45}$ argue that personal control may not always be necessary and that alignment with powerful individuals, groups or institutions (such as AA) may serve to enhance control among some individuals (Weisz, et al. refer to this as "secondary control"). The utility of this type of control may depend upon perceptions that the powerful entity is, indeed, effective; the length of affiliation the powerful entity offers the client (short affiliations might leave the client stranded); and the locus of control of the client (powerful others may be more important to those with external locus of control).

Many, if not most, clinic-based treatment programs for changing health behaviors adopt these traditional methods of change, probably because they reflect strategies 
often successfully employed for physical conditions. Relegating poor health practices to the status of "diseases" obligates persons wishing to change their lifestyles to seek outside treatment from an expert. The treatment program, then, shifts the locus of responsibility in changing the desirable behavior from the client to the therapist; from the efficacy of the individual to the efficacy of the treatment.

However, despite the medicalization of many poor health-related practices, the vast majority of modifications in drug use, obesity and cigarette smoking are accomplished without external treatment. ${ }^{46.47}$ Chapman $^{48}$ compellingly argues that treating smoking as a behavior requiring intensive external treatment tends to heighten smokers' perceptions that their behavior is, indeed, difficult or impossible to change on their own. Chapman states: "Stopping smoking is seen as a cure, and if smoking needs to be cured, then the magical, laying-on-of-hands accoutrements of curing must parallel that of medicine in general. Clinics and groups are tangible entities, located in buildings with staff who have concrete, comprehensible tasks, unlike most health education work that sounds vague and has imperceptible effects in the short term . . . The mystique and paraphernalia of popular notions of psychology, accupuncture, and hypnotism are infinitely more newsworthy than the fact that most people stop smoking unspectacularly and alone. There is the expectation that stopping is extremely difficult and that one should place oneself passively in the hands of a healer." (p.919) Programs which employ self-help modes or minimal therapist contact in conjunction with self-help may present behavior change strategies in a way that removes the mysticism of change and better promotes personal coping skills and enhances efficacy. ${ }^{49}$

These arguments are not meant to imply that all self-help measures for modifying health behaviors will enhance efficacy, or that self-help is the only mode for enhancing efficacy. Nor are they meant to suggest that all clinic-based programs do not enhance efficacy. Many self-help programs to change health behavior give the person little freedom in choosing change methods, do not reward stages of change (only complete change) and are extremely complicated and wordy-implying the presence of an "expert" in the background. Conversely, many clinic-based programs place the onus of responsibility for change on the individual, rewarding and encouraging stages of success. Efficacy is then often enhanced through a series of performance accomplishments, which motivate attempts to take on more difficult tasks.

With regard to maintenance of behavior. traditional models of change tend to equate a single retum to an undesirable behavior (e.g., taking a drink, smoking a cigarette) with relapse and attribute relapse to physiological factors - assuming that the individual has little control over relapse and equating it with treatment failure. ${ }^{41}$ However, as Marlatt states, whether a lapse leads to total return to the undesired behavior depends largely on the individual's efficacy expectations. Low efficacy may lead a person undergoing a lapse in behavior to attribute the lapse to personal weakness, thereby reducing chances of recovering from such an event ${ }^{50}$ Studies reviewed in this paper (e.g., McIntyre, et al..$^{15}$ Jeffery, et al. ${ }^{26}$ ) support the view that high levels of selfefficacy are required to maintain change.

To summarize, this review found a consistently-positive relationship between selfefficacy and health behavior change and maintenance. Many programs designed to influence health practices implicitly enhance efficacy expectations. These programs may be improved through directly targeting the enhancement of self-efficacy. However, programs which use traditional methods of treating patients may diminish, rather than enhance, efficacy. A better understanding of the circumstances in which self-efficacy 
is important and strategies for enhancing efficacy in practice should lead to more effective health promotion programs for a greater number of people.

The authors wish to thank Dr. Kenneth McLeroy, Dr. Lynda Anderson and Susan Blalock for their helpful comments.

\section{References}

1. Bandura A: Social Learning Theory. New Jersey, Prentice Hall, 1977

2. Bandura A: Self-efficacy: Toward a unifying theory of behavioral change. Psychol Rev 84:191-215, 1977.

3. Bandura A: Self-efticacy mechanism in human agency. Am Psychol 37:122-147, 1982.

4. Bandura A: The self and mechanisms of agency, in Suls J (ed): Psychological Perspectives on the Self Vol I. Hillsdalc. NJ. Erlbaum. 1982.

5. Schunk D. Carbonari J: Self-efticacy models, in Matarazzo J, Weiss S, Herd J, Miller N, Weiss $S$ (eds): Behavioral Health: A Handbook of Health Enhancement and Disease Prevention. New York, NY. John Wiley \& Sons. 1984

6. Bandura A: Recycling misconceptions of perceived self-efficacy. Cog Ther Res 8:231-255, 1984.

7. Wallston BS, Waltston KA: Social Psychological models of health behavior: An examination and integration, in Baum A. Taylor Sc. Singer JE (eds): Handbook of Psychology and Health Vol IV. Hillsdale. NJ. Erlbaum, 1984.

8. Crandall R: The measurement of self-esteem and related constructs, in Robinson JP. Shaver PR (eds): Measures of Social Psychological Altindes. Ann Arbor. MI. Institute for Social Research, 1978.

9. Lazarus RS. Folkman S: Siress, Appruisal and Coping. New York. Springer Publishing, 1984.

10. Abramson LY. Garber J. Seligman MEP: Learned helplessness in humans: An attributional analysis, in Garber J. Seligman MEP (eds): Human Helplessness: Theory and Applications. NY. Academic Press, 1980.

11. Pechacek TGF. Danaher BG: How and why people quit smoking: A cognitive-behavioral analysis, in Kendall PC. Hollow SD (eds): Cognitive-Behavioral Interventions: Theory, Research, and Procedures. New York. Academic Press. 1979.

12. Prochaska JO. DiClemente CC: Self change processes, self-efficacy and decisional balance across five stages of smoking cessation, in Liss AR (cd): Adrances in Cancer Control: Epidemiology and Research. New York, Alan R. Liss, Inc., 1984.

13. Brod MI. Hall SM: Joiners and nonjoiners in smoking treatment: A comparison of psychosocial variables. Addict Beha: 9:217-221, 1984.

14. Condiotte MM, Lichtenstein E: Self-efficacy and relapse in smoking cessation program. J Consult Clin Psychol 49:648-658, 1981.

15. McIntyre, DO. Lichtenstein E, Mermelstein RJ: Self-cfficacy and relapse in smoking cessation: A replication and extension. J Consult Clin Psychol 51:632-633, 1983.

16. DiClemente CC: Self-efficacy and smoking cessation maintenance: A preliminary report. Cog Ther Res 5:175-187. 1981

17. Coelho RJ: Self-efficacy and cessation of smoking. Psychol Rep 54:309-310. 1984.

18. Godding PR, Glasgow RE: Self-efficacy and outcome expectations as predictors of controlled smoking status. Cog Ther Res, (in press).

19. Strecher VJ, Becker MH, Kirscht JP, Eraker SA, Graham-Tomasi RP: Psychosocial aspects of changes in cigarette-smoking behavior. Patient Educ Couns 7:249-262, 1985.

20. Seligman MEP: Helplessness. San Francisco, Freeman, 1975.

21. Maddux JE, Rogers RW: Protection motivation and self-efficacy: A revised theory of fear appeals and attitude change. J Exp Soc Psychol 19:469-479. 1983.

22. Nicki RM, Remington RE, MacDonald GA: Self-efficacy, nicotine-fading/self-efficacy monitoring and cigarette smoking behavior. Behan Res Ther 22:477-485. 1985.

23. Chambliss CA. Murray EJ: Cognitive procedures for smoking reduction: Symptom attribution versus efficacy attribution. Cog Ther Res 3:91-95. 1979.

24. Rotter JB: Generalized experiences for intemal versus external control of reinforcement. Psychol Monogr 80:1014-1053, 1966

25. Blittner M. Goldberg J, Merbaum M: Cognitive self-control factors in the reduction of smoking behavior Behav Ther 9:553-561, 1978.

26. Jeffrey RW, Bjornson-Benson WM, Rosenthal BS, Lindquits RA, Kurth CL, and Johnson SL: Correlates of weight loss and its maintenance over two years of follow-up among middle-aged men. Prev Med 13:155-168, 1984. 
27. Chambliss CA. Murray EJ: Efficacy attribution: Locus of control, and wcight loss. Cog Ther Res 3:349-353, 1979 .

28. Gilchrist LD, Schinke SP: Coping with contraception: Cognitive and behavioral methods with adolescents. Cog Ther Res 7:379-388. 1983.

29. McKinney, K: Perceptions of efficacy and control, and contraceptive behavior. Unpublished Doctoral Dissertation. University of Wisconsin, Madison, Wisconsin, 1982.

30. Levinson RA: Teenage women and contraceptive hehavior: Focus on self-eflicacy in scxual and contraceptive siluations. Unpublished Doctoral Dissertation. Stanford University. Palo Alto, California. 1982.

31. Rist F, Watzl H: Self assessment of relapse risk and assertiveness in relation to treatment outcome of female alcoholics. Addict Behav 8:121-127. 1983.

32. Weinberg RS. Gould D. Jackson A: Expectations and performance: An empirical test of Bandura's self-efficacy theory. I Sport Psichol 1:320-331. 1979.

33. Ewart CK. Taylor CB. Recse LB. Debusk RF: Effects of early postmyocardial infarcation exercise testing on self-perception and subsequent physical activity. Am J Cardiol 41:1076-1080, 1984

34. Kaplan RM. Atkins CJ. Reinsch S: Specitic efficacy expectations media exercise compliance in patients with COPD. Health Psychol 3:223-242, 1984.

35. Becker $\mathrm{MH}$ (ed): The health belief model and personal health hehavior. Healh Educ Monogr 2:324-508. 1974.

36. Janz NK. Becker MH: The health belief model: A decade later. Health Edwc $Q$ 11:1-47. 1984.

37. Kirsch 1: Efficacy expectations or response predictions: The meaning of efficacy ratings as a function of task charactcristics. J Pers Soc Psichol 42:132-136. 1982.

38. Kirsch I: Self-efficacy and expectancy: Old wine with new labels. J Pers Soc Psychol 49:824-830. 1985.

39. Bandura A: Self-efticacy determinants of anticipated fears and calamities. J Pers Soc Prychol 45:464-469. 1983.

40. Baer JS, Holt CS, Lichtenstein E: Self-efficacy and smoking re-examined: Construct validity and clinical validity. (in press).

41. Marlatt GA. Gurdon JR (cds): Relapse Prevention: Maintenance Strategies in the Treatment of Addictive Behaviors. New York. Guilford Press, 1985

42. Bartlett E: Educational self-help approaches in childhood asthma. J Allergy Clin Immunol 72:545-553, 1983.

43. Brickman P. Redinuwitz VC. Kawzu J, Coates J. Cohn E, Kidder L: Models of helping and coping. Am Psuchol 37:368-384, 1982.

44. Clifford JS: Self-efficacy counseling and the mainkenance of sobriety. Pers Guid J 62:111-114, 1983.

45. Weisz. JR. Rothhaum FM. Blackbum TC: Standing out and standing in: The psychology of control in America and Japan. Am Psychol 39:955-969, 1984.

46. Hamburg DA. Elliott GR. Parson DL (eds): Healh Behavior: Fromiers of Research in the Biobeharioral Sciences. Washington. D.C.. National Academy Press. 1982.

47. Schacter S: Recidivism and self-care of smoking and obesity. Am Psychol 37:436-444, 1982.

48. Chapman S: Stop smoking clinics: A case for their abandonment. Lancet 1:918-920. 1985.

49. Strecher VJ: A minimal-contact smoking cessation program for a health care setting. Public Health Rep 50:497-502, 1983.

50. Brownell KD. Marlatt GA. Lichtenstein E. Wilson GT: Understanding and preventing relapse. Am Psichol, (in press).

51. Prochaska JO. Crimi P. Lapanski D. Mantel L. Reid P: Self-change processes, self-cfficacy and selfconcept in relapse and maintenance of cessation of smoking. Psychol Rep 51:983-990, 1982. 